\title{
Solution of the Local Mass Problem in General Relativity
}

\author{
Yvonne Choquet-Bruhat \\ Département de Mécanique, Université de Paris VI, F-Paris, France \\ Jerrold E. Marsden ${ }^{\star}$ \\ Department of Mathematics, University of California, Berkeley, California 94720, USA
}

\begin{abstract}
The local mass problem is solved. That is, in suitable function spaces, it is shown that for any vacuum space-time near flat space, its mass $m$ is strictly positive. The relationship to other work in the field and some discussion of the global problem is given. Our proof is, in effect, a version of critical point analysis in infinite dimensions, but detailed $L^{p}$ and Sobolev-type estimates are needed for the precise proof, as well as careful attention to the coordinate invariance group. For the latter, we prove a suitable slice theorem based on the use of harmonic coordinates.
\end{abstract}

\section{Introduction}

For some time there has been controversy over the definition and positivity of the mass of an asymptotically euclidean (vacuum) solution of Einstein's equations.

Brill [3] established positivity for time-symmetric and axial-symmetric spacetime. Araki [1] proved the positivity of the second variation of the Schwarzchild mass of a certain class of time-symmetric solutions constructed by conformal methods. Finally Brill and Deser [4] outlined a proof of positivity in the general case.

The method of Brill and Deser is to show that the mass function has only one critical point, namely at flat space and that the second variation is strictly positive there. The proof is, however, incomplete for four reasons.

First of all, they assumed the existence of maximal slices (i.e., slices whose second fundamental form has zero trace), which was open to question. Secondly, the topology in which the second variation is positive definite is not the same as the topology on the initial data set, so that it is far from obvious that flat space is a local minimum. Thirdly, the problems connected with the coordinate invariance group require attention on the space of initial data as well as on an infinitesimal level (the quotient space may well be singular). Fourthly, the global

* Partially supported by the University of Toronto, Université de Paris VI and NSF Grant MPS75-05576 
assertion that a real valued function with a single non-degenerate critical point which is a local minimum has that point as a global minimum is not true. Counterexamples are easly constructed on $\mathbb{R}^{2}$.

These problems have been pointed out by several authors. For example, Geroch [20] has a summary of what is known. O'Murchadha and York [22] have speculated that, because of the first difficulty, negative (and therefore arbitrarily large negative) mass might exist. Similar problems with the Bondi mass have been pointed out by Robinson and Winicour [27].

Recently, new interest in the mass problem has been sparked by the proof that maximal slices do exist (Choquet-Bruhat [12], Cantor et al. [10]). This shows that the first difficulty above may be eliminated.

We can divide the mass problem into two parts corresponding to the second and third and the third and fourth difficulties:

(i) local problem: is the mass positive near flat space?

(ii) global problem: is the mass positive for a spacetime that can be connected to flat space?

Up until now, neither question has been answered in rigorous fashion. The purpose of this paper is to show that (i) has an affirmative answer. We shall also make some additional remarks on (ii).

Our proof consists of two parts. We must reduce things to a nondegenerate situation by eliminating the coordinate invariance. For this we construct a local slice for the action near flat space, using harmonic coordinates. Secondly we must show that on this space, flat space is a local minimum by making some estimates. Our procedures are inspired by the use of weak Riemannian structures in $[15,16]$ and Tromba's Morse theory for weak Riemannian structures $[28,29]$.

We wish to thank J. Arms, A. Fischer, R. Sachs, A. Taub, A. Tromba and A. Weinstein for several useful remarks.

\section{Notation and Function Spaces}

For $1<p<\infty, \delta \in \mathbb{R}$ and $s \in \mathbb{Z}^{+}$, let $M_{s, \delta}^{p}\left(\mathbb{R}^{n}, \mathbb{R}^{m}\right)$ be the completion of the $C^{\infty}$ functions $f: \mathbb{R}^{n} \rightarrow \mathbb{R}^{m}$ with compact support using the norm

$$
\|f\|_{p, s, \delta}=\sum_{0 \leqq \alpha \leqq s}\left\|\sigma^{\delta+\alpha} D^{\alpha} f\right\|_{L^{p}}
$$

where $\sigma(x)=\left(1+|x|^{2}\right)^{1 / 2}$ and $D^{\alpha} f$ is the (total) derivative of $f$ of order $\alpha$.

Note that if $f \in M_{s, \delta}^{p}$ then $D f \in M_{s-1, \delta+1}^{p}$.

These spaces were introduced and studied by Nirenberg-Walker [24] and Cantor $[6,7]$. Various properties of these spaces, proved in these references, will be needed. For example:

1. If $p>n /(n-2), \delta=0$ and $s \geqq 2$, then the Laplacian $\Delta: M_{s, \delta}^{p} \rightarrow M_{s-2, \delta+2}^{p}$ is an isomorphism (onto).

2. If $p>1, s>n / p, \delta \geqq 0$ and $0 \leqq l \leqq s$, then multiplication

$$
M_{s, \delta}^{p} \times M_{s-l, \delta+l}^{p} \rightarrow M_{s-l, \delta+l}^{p}
$$

is continuous bilinear.

3. If $s>n / p+k$, then $M_{s, \delta}^{p} \subset C^{k}$, with the inclusion continuous. 
For purposes of this paper we shall choose $n=3, \delta=0,3<p<6$ and $s \geqq 3$. The reason for $p>3$ is so that property 1 holds. The reason for $p<6$ is contained in Lemma 1.3 below.

On $\mathbb{R}^{3}, f \in M_{s, \delta}^{p}$ has the intuitive meaning that $f \sim \frac{1}{r}, D f \sim \frac{1}{r^{2}}, \ldots, D^{s} f \sim$ $\frac{1}{r^{s+1}}$ at $\infty$, provided $p>3, \delta=0$. In particular, note that for such an $f, f \notin L_{2}$ but it is reasonable that $D f \in L_{2}$.

Let $\gamma$ denote the standard euclidean metric on $\mathbb{R}^{3}$ and let $\mathscr{M}_{s, \delta}^{p}$ denote the set of Riemannian (positive definite) metrics $g$ such that $g-\gamma \in M_{s, \delta}^{p}$. Then $\mathscr{M}_{s, \delta}^{p}$ is an open cone in $S_{s, \delta}^{p}+\{\gamma\}$, where $S_{s, \delta}^{p}$ denotes the Banach space of symmetric 2-tensors of class $M_{s, \delta}^{p}$. Thus, the tangent space to $\mathscr{M}_{s, \delta}^{p}$ at $g$ is $T_{g} \mathscr{M}_{s, \delta}^{p}=S_{s, \delta}^{p}$.

Let $I: \mathbb{R}^{3} \rightarrow \mathbb{R}^{3}$ be the identity map and let $\mathscr{D}_{s, \delta}^{p}$ denote the diffeomorphisms $\eta$ of $\mathbb{R}^{3}$ such that $\eta-I$ and $\eta^{-1}-I$ are of class $M_{s, \delta}^{p}$. If $s>\frac{n}{p}+1, \delta \geqq 0$, then $\mathscr{D}_{s, \delta}^{p}$ is a smooth manifold and a topological group (Cantor [7]).

It is convenient to enlarge $\mathscr{D}_{s+1, \delta-1}^{p}$ somewhat. Let $\overline{\mathscr{D}}_{s+1, \delta-1}^{p}$ denote those diffeomorphisms $\eta$ of $\mathbb{R}^{3}$ such that $d \eta$ and $d \eta^{-1}$ are of class $M_{s, \delta}^{p}$. Again, $\overline{\mathscr{D}}_{s+1, \delta-1}^{p}$ is a topological group and a smooth manifold if $s>\frac{n}{p}$ and $\delta \geqq 0$. This and the following can be proved as in Cantor [7] (see Ebin [15] for the case of compact manifolds instead of $\mathbb{R}^{n}$ ).

1.1. Lemma. $\overline{\mathscr{D}}_{s+1, \delta-1}^{p}$ acts continuously on $\mathscr{M}_{s, \delta}^{p}$ by pull-back: $(\eta, g) \mapsto \eta^{*} g$.

This uses the multiplication property 2 . above and the fact that composition

$$
M_{s, \delta}^{p} \times \overline{\mathscr{D}}_{s, \delta-1}^{p} \rightarrow M_{s, \delta}^{p} ;(f, \eta) \mapsto f \circ \eta
$$

is continuous.

We remark that the orbit $\mathcal{O}_{\gamma}$ of flat space need not be a submanifold of $\mathscr{M}_{s, \delta}^{p}$. If we were to let $\overline{\mathscr{D}}_{s+1, \delta}^{p}$ act on $\mathscr{M}_{s, \delta+1}^{p}, \mathcal{O}_{\gamma}$ would be a submanifold but $\mathscr{M}_{s, \delta+1}^{p}$ has too strong asymptotic behavior to be useful for present purposes.

1.2. Lemma. If $\sigma f \in L_{p}\left(\mathbb{R}^{n}\right)$, then $f \in L_{r}$ provided $p \geqq r>3 p /(p+3)$.

Proof. By Holder's inequality,

$$
\int|f|^{r} d x \leqq\left(\int|\sigma|^{p} d x\right)^{1 / q} \cdot\left(\int \sigma^{-r q^{\prime}} d x\right)^{1 / q^{\prime}}
$$

where $q=p / r, \frac{1}{q}+\frac{1}{q^{\prime}}=1$. The last integral is finite if $r q^{\prime}=r p /(p-r)>3$; i.e., if $r>3 p /(p+3)$.

1.3. Lemma. If $2 \leqq p<6, \delta=0$ and $s \geqq 2$, then $f \in M_{s, \delta}^{p}$ implies $f \in L$ and $D f \in L_{2}$. In fact, if we write $\|f\|_{E}^{2}=\|D f\|_{L_{2}}^{2}$ (the "energy norm"), we have inequalities

$$
\|f\|_{L^{6}} \leqq \text { (Constant) }\|f\|_{E} \leqq \text { (Constant) }\|f\|_{p, s, \delta} .
$$


Proof. If $f$ is in some $L_{q}$ space, we have the inequality $\|f\|_{L^{6}} \leqq C\|D f\|_{L^{2}}$ (see Nirenberg [23]). Thus we need only show $D f \in L^{2}$. However, $\sigma D f \in L_{p}$ so by Lemma 1.2, $D f \in L_{r}, p \geqq r>3 p /(p+3)$. For $p<6,3 p /(p+3)<2$, so $r=2$ can be chosen.

\section{The Mass Function and Its First Derivative}

Define $\bar{m}: \mathscr{M}_{s, \delta}^{p} \times S_{s-1, \delta+1}^{p} \rightarrow \mathbb{R}$ by

$$
\begin{aligned}
16 \pi \bar{m}(g, k) & \left.=\oint_{\infty}\left(\partial_{j} g_{i j}-\partial_{i} g_{j j}\right) d S^{i}-\int_{\mathbb{R}^{3}}\left(R(g)-k \cdot k+(\operatorname{tr} k)^{2}\right) d \mu(g)\right) \\
& =\int_{\mathbb{R}^{3}}\left(\partial_{i} \partial_{j} g_{i j}-\partial_{i} \partial_{i} g_{j j}\right) d^{3} x-\int_{\mathbb{R}^{3}}\left(R(g)-k \cdot k+(\operatorname{tr} k)^{2}\right) d \mu(g) .
\end{aligned}
$$

Here, $\oint_{\infty}$ denotes the surface integral at infinity in euclidean coordinates, $R(g)$ is the scalar curvature of $g, \operatorname{tr} k=\operatorname{tr}_{g} k$ is the trace of $k$ with respect to the metric $g$ and $d \mu(g)$ is the volume form of $g$. One may alternatively write

$$
16 \pi \bar{m}(g, k)=\int_{\mathbb{R}^{3}} D R(\gamma) \cdot(g-\gamma) d \mu(\gamma)-\int_{\mathbb{R}^{3}}\left(R(g)-k \cdot k+(\operatorname{tr} k)^{2}\right) d \mu(g)
$$

where $D R(\gamma) \cdot h$ is the derivative of $R(g)$ at $\gamma$ in direction $h ; R: \mathscr{M}_{s, \delta}^{p} \rightarrow M_{s-2, \delta+2}^{p}$ is a $C^{\infty}$ mapping with derivative $D R(g) \cdot h=-\Delta(\operatorname{tr} h)+\delta \delta h-\operatorname{Ric}(g) \cdot h$ where $\Delta=\Delta_{g}=\nabla_{i} \Delta^{i}$ is the Laplacian and $(\delta h)_{j}=\left(\delta_{g} h\right)_{j}=\nabla_{i} h_{j}^{i}$ is the divergence - cf. Lichnerowicz [21] and Fischer-Marsden [18]. Notice that

$$
\int_{\mathbb{R}^{3}} D R(\gamma) \cdot h d \mu(\gamma)=\int_{\mathbb{R}^{3}} D R(g) \cdot h d \mu(g)+\int_{\mathbb{R}^{3}} \operatorname{Ric}(g) \cdot h d \mu(g)
$$

since $g$ is asymptotically equal to $\gamma$.

As remarked by DeWitt [14] and emphasized by Regge and Teitelboim [26], $\bar{m}(g)$ is the numerical value of the "real" Hamiltonian which generates the dynamical Einstein equations.

It is not hard to check that $\bar{m}$ is a well defined $C^{\infty}$ mapping, using our choices of $p, s$ and $\delta$. On the constraint submanifold of relativity, $\bar{m}$ coincides with the usual formula for $m$ in Brill and Deser [4]. (See Choquet-Bruhat and Deser [13] and Fischer and Marsden $[18,19]$ for the proof that the constraint set near $\gamma$ is actually a $C^{\infty}$ manifold.) Also, $m$ and $\bar{m}$ are invariant under the action of $\overline{\mathscr{D}}_{s+1, \delta-1}^{p}$.

The first and second derivatives of $\bar{m}$ can be calculated in a straightforward manner using the formulas for the derivatives of $R(g)$ and $\operatorname{Ric}(g)$ (the Ricci tensor) from Lichnerowicz [21]. One obtains

$$
\begin{aligned}
& 16 \pi d \bar{m}(g, k) \cdot(h, w) \\
& =\int_{\mathbb{R}^{3}}\left\{\operatorname{Ric}(g)-\frac{g}{2}\left(R(g)-k \cdot k+(\operatorname{tr} k)^{2}\right)\right\} \cdot h d \mu(g) \\
& \quad+2 \int_{\mathbb{R}^{3}}(k-g \operatorname{tr} k) \cdot w d \mu(g)
\end{aligned}
$$

for the derivative of $\bar{m}$ in the direction $(h, w) \in S_{s, \delta}^{p} \times S_{s-1, \delta+1}^{p}$ at the point $(g, k)$.

Observe that in (2.2) there are no constraints or trace conditions used.

From (2.2) it is obvious that $(g, k)$ is a critical point of $\bar{m}$; i.e., $d \bar{m}(g, k)=0$, if and only if $k-g \operatorname{tr} k=0$ (i.e., $k=0$ ) and $\operatorname{Ric}(g)-\frac{g}{2} R(g)=0$ (i.e., $g$ is flat). 
Let

$$
\begin{aligned}
\mathscr{C}= & \left\{(g, k) \in \mathscr{M}_{s, \delta}^{p} \times S_{s-1, \delta+1}^{p} \mid R(g)-k \cdot k+(\operatorname{tr} k)^{2}=0\right. \\
& \text { and } \delta(k-g(\operatorname{tr} k))=0\}
\end{aligned}
$$

be the constraint manifold. Then the tangent space to $\mathscr{C}$ at $(g, k)$ is given by

$$
\begin{gathered}
T_{(g, k)} \mathscr{C}=\{(h, w) \mid-\Delta(\operatorname{tr} h)+\delta \delta h-\operatorname{Ric}(g) \cdot h-2(k-g \operatorname{tr} k) \cdot w=0 \\
\text { and } \left.\delta w-d \operatorname{tr} w+\frac{1}{2} k \cdot \nabla h-\frac{1}{2} k \cdot d \operatorname{tr} h=0\right\} .
\end{gathered}
$$

Since $\bar{m}$ is invariant under the action of $\overline{\mathscr{D}}_{s+1, \delta}^{p}, d \bar{m}(g, k) \cdot(h, w)=0$ if $(h, w)$ is tangent to the orbit of $(g, k)$ under $\overline{\mathscr{D}}_{s+1, \delta}^{p}$.

By a theorem of O'Murchadha and York [22], which can be easily proved in our $M_{s, \delta}^{p}$ context, to every asymptotically euclidean spacetime satisfying the weak energy condition admitting a maximal slice (i.e., one whose second fundamental form has zero trace), there is a vacuum spacetime admitting an instant of time symmetry and smaller, or equal mass. Thus, we can restrict outselves to the case $k=0$, since maximal slices exist for spaces satisfying the strong energy condition $([12,10])$.

Define

$$
\bar{m}: \mathscr{M}_{s, \delta}^{p} \rightarrow \mathbb{R},
$$

by

$$
16 \pi \bar{m}(g)=\oint_{\infty}\left(\partial_{j} g_{i j}-\partial_{i} g_{j j}\right) d S_{i}-\int_{\mathbb{R}^{3}} R(g) d \mu(g)
$$

so that

$$
16 \pi d \bar{m}(g) \cdot h=\int_{\mathbb{R}^{3}}\left(\operatorname{Ric}(g)-\frac{g}{2} R(g)\right) \cdot h d \mu(g), h \in S_{s, \delta}^{p} .
$$

The constraint set becomes $\mathscr{C}=\left\{g \in \mathscr{M}_{s, \delta}^{p} \mid R(g)=0\right\}$, which is $([13,18])$ a manifold with tangent space consisting of $h \in S_{s, \delta}^{p}$ satisfying

$$
-\Delta(\operatorname{tr} h)+\delta \delta h-\operatorname{Ric}(g) \cdot h=0 \text {. }
$$

Note that if $g \in \mathscr{C}$, i.e. $R(g)=0$ holds, then

$16 \pi d \bar{m}(g) \cdot h=\int_{\mathbb{R}^{3}} \operatorname{Ric}(g) \cdot h d \mu(g)$.

Again, let $m$ be the restriction of $\bar{m}$ to $\mathscr{C}$, so (2.8) holds for $m$.

2.1. Lemma. $g \in \mathscr{M}_{s, \delta}^{p}$ is a critical point of $m$ if and only if $\operatorname{Ric}(g)=0$.

Proof. Suppose $g$ is a critical point of $m$. Then

$$
\int_{\mathbb{R}^{3}} \operatorname{Ric}(g) \cdot h d \mu(g)=0
$$

for all $h \in T_{g} \mathscr{C}$. For $k \in S_{s, \delta}^{p}$ arbitrary, let

$$
h=k-\frac{1}{3} g \operatorname{tr} k+\frac{1}{3} g \tau
$$

where $\tau$ is defined by

$$
\frac{2}{3} \Delta \tau=\delta \delta k-\frac{1}{3} \Delta \operatorname{tr} k-\operatorname{Ric}(g) \cdot k .
$$


A unique such $\tau \in M_{s, \delta}^{p}$ exists, since $\Delta: M_{s, \delta}^{p} \rightarrow M_{s-2, \delta+2}^{p}$ is an isomorphism. Then one checks easily that $h$ given by (2.9) satisfies (2.7); $\operatorname{tr} h=\tau$, and $\delta \delta h=$ $\delta \delta k-\frac{1}{3} \Delta(\operatorname{tr} h)+\frac{1}{3} \Delta \tau$. Moreover,

$$
\int_{\mathbb{R}^{3}} \operatorname{Ric}(g) \cdot h d \mu(g)=\int_{\mathbb{R}^{3}} \operatorname{Ric}(g) \cdot k d \mu(g) .
$$

Since $R(g)=0$; this vanishes for all $k \in S_{s, \delta}^{p}$ only if $\operatorname{Ric}(g)=0$ and so the lemma follows.

2.2. Lemma. If $s \geqq 3, g \in \mathscr{M}_{s, \delta}^{p}$ and $\operatorname{Ric}(g)=0$, then $g \in \mathcal{O}_{\gamma}$, the orbit of flat space under $\overline{\mathscr{D}}_{s+1, \delta-1}^{p}$ (see Lemma 1.1).

Proof. Since $g$ is flat and asymptotically euclidean, its exponential map will be a diffeomorphism. As in Fischer-Marsden [18], §4, this map will be of class $\overline{\mathscr{D}}_{s+1, \delta-1}^{p}$. (See also Lemma 5.2 below.) It follows that $g \in \mathcal{O}_{\gamma}$.

\section{The Second Derivative of $\bar{m}$}

From now on, assume $s \geqq 3, \delta=0$ and $3<p<6$, and recall from Lemma 1.3 that if $f \in M_{s, \delta}^{p}$ then $f \in L_{6}$ and $D f \in L_{2}$.

3.1. Lemma. The second derivative of $\bar{m}$ is given by

$$
\begin{aligned}
16 \pi d^{2} \bar{m}(g)(h, h)= & \frac{1}{2} \int_{\mathbb{R}^{3}} \nabla h \cdot \nabla h d \mu(g)+\int_{\mathbb{R}^{3}}\left\{-\frac{1}{2}(d \operatorname{tr} h)^{2}\right. \\
& -(\delta h)^{2}-(d \operatorname{tr} h) \cdot \delta h-(\operatorname{tr} h)(\operatorname{Ric}(g) \cdot h) \\
& +\operatorname{Ric}(g) \cdot h \times h\} d \mu(g) \\
& +\int_{\mathbb{R}^{3}}\left\{\frac{1}{4} R(g)(\operatorname{tr} h)^{2}-R(g)(h \cdot h)\right\} d \mu(g)
\end{aligned}
$$

where $\cdot$ is contraction of tensors and $(h \times h)_{i j}=h_{i l} h_{j}^{l}$.

Proof. Again, a straightforward though tedious computation.

If $\operatorname{Ric}(g)=0, \operatorname{tr} h=0$ and $\delta h=0,(3.1)$ reduces to (twice the square of) the energy norm for the inner product

$$
\langle h, k\rangle_{g}=\int_{\mathbb{R}^{3}} \nabla h \cdot \nabla k d \mu(g) .
$$

We already know this is well defined; see Lemma 1.3.

As in Ebin [15], p. 19, one proves the following (using the properties 1 and 2 of $M_{s, \delta}^{p}$ spaces from $\S 1$ ).

3.2. Lemma. $\langle,\rangle_{g}$ defines on $\mathscr{M}_{s, \delta}^{p}$, a smooth weak Riemannian structure which has a smooth connection $\nabla$.

As noted in 1.3, we shall let \|\|$_{E}$ denote the energy norm in the Euclidean metric; $\|h\|_{E}^{2}=\langle h, h\rangle_{\gamma}=\int_{\mathbb{R}^{3}}\left|\partial_{l} h_{i j}\right|^{2} d^{3} x$.

\section{Local Positivity of $m$ in a Slice}

Let $S=\left\{g \in \mathscr{M}_{s, \delta}^{p} \mid g^{i j} \Gamma_{i j}^{k}=0\right\}$; i.e., metrics for which euclidean coordinates are harmonic. This condition arises in the study of the initial value problem for general relativity (Choquet-Bruhat [11]). 
4.1. Lemma. $S \subset \mathscr{M}_{s, \delta}^{p}$ is a $C^{\infty}$ submanifold in a neighborhood of $\gamma$. Furthermore, $S$ is modelled on the Banach space

$$
E_{1}=\left\{h_{1} \in S_{s, \delta}^{p} \mid \delta_{\gamma} h_{1}-\frac{1}{2} d \operatorname{tr}_{\gamma} h_{1}=0\right\} .
$$

Proof. We first show that $S_{s, \delta}^{p}$ splits as a $\langle,\rangle_{\gamma}$ orthogonal topological sum

$$
S_{s, \delta}^{p}=E_{1} \oplus E_{2}
$$

where $E_{2}=\left\{\left(h_{2}\right)_{i j}=-\frac{1}{2} \Delta_{\gamma}^{-1}\left(\partial_{i} u_{j}+\partial_{j} u_{i}\right)+\frac{1}{2}\left(\Delta_{\gamma}^{-1} \partial_{l} u_{l}\right) \delta_{i j} \mid u_{j} \in M_{s-1, \delta+1}^{p}\right\} \subset S_{s, \delta}^{p}$.

Indeed, let $h \in S_{s, \delta}^{p}$. Let $a_{j}=\partial_{i} h_{i j}-\frac{1}{2} \partial_{j} h_{l l} \in M_{s-1, \delta+1}^{p}$ and let $u_{j}=-2 a_{j}+$ $\frac{2}{3} \partial_{j} \Delta_{\gamma}^{-1} \partial_{i} a_{i}$. Note that $\partial_{i} u_{i}=-\frac{4}{3} \partial_{j} a_{j}$ since $\Delta_{\gamma}=\partial_{i} \partial_{i}$ and $\partial_{j}$ and $\Delta_{\gamma}^{-1}$ commute since we are at flat space. $\left(\nabla_{i}\right.$ and $\Delta_{g}^{-1}$ do not, in general, commute for $g \neq \gamma$.) Define $h_{2}$ as above and let $h_{1}=h-h_{2}$. Then $h_{1} \in E_{1}$ since

$$
\begin{aligned}
\delta_{\gamma} h_{1}-\frac{1}{2} d \operatorname{tr}_{\gamma} h_{1}= & \partial_{i} h_{i j}-\frac{1}{2} \partial_{j} h_{i i}-\partial_{i}\left(h_{2}\right)_{i j}+\frac{1}{2} \partial_{j}\left(h_{2}\right)_{i i} \\
= & \partial_{i} h_{i j}-\frac{1}{2} \partial_{j} h_{i i}+\frac{1}{2} \partial_{i} \Delta_{\gamma}^{-1}\left(\partial_{i} u_{j}+\partial_{j} u_{i}\right) \\
& -\frac{1}{2} \partial_{i} \Delta_{\gamma}^{-1}\left(\partial_{l} u_{l}\right) \delta_{i j}-\frac{1}{4} \partial_{j} \Delta_{\gamma}^{-1}\left(\partial_{i} u_{i}+\partial_{i} u_{i}\right) \\
& +\frac{1}{4} \partial_{j}\left(\Delta_{\gamma}^{-1} \partial_{l} u_{l}\right) \delta_{i i} \\
= & a_{j}+\frac{1}{2} u_{j}-\frac{2}{3} \partial_{j} \Delta_{\gamma}^{-1} \partial_{i} a_{i}+\frac{2}{3} \partial_{j} \Delta_{\gamma}^{-1} \partial_{i} a_{i} \\
& +\frac{2}{3} \partial_{j} \Delta_{\gamma}^{-1} \partial_{i} a_{i}-\frac{3}{4} \cdot \frac{4}{3} \partial_{j} \Delta_{\gamma}^{-1}\left(\partial_{i} a_{i}\right) \\
= & a_{j}+\frac{1}{2} u_{j}-\frac{1}{3} \partial_{j} \Delta_{\gamma}^{-1} \partial_{i} a_{i}=0
\end{aligned}
$$

by definition of $u_{j}$.

If $h_{1} \in E_{1} h_{2} \in E_{2}$, and $L_{u}$ denotes Lie differentiation,

$$
\begin{aligned}
\left\langle h_{1}, h_{2}\right\rangle_{\gamma} & =\int \nabla h_{1} \cdot \nabla h_{2} d \mu(g) \\
& =-\int h_{1} \Delta_{\gamma} h_{2} d x=-\int\left\{-\frac{1}{2} h_{1} \cdot L_{u} \gamma+\frac{1}{2} \operatorname{tr}_{\gamma} h_{1} \delta u\right\} d x \\
& =-\int\left(\delta_{\gamma} h_{1}-\frac{1}{2} d \operatorname{tr}_{\gamma} h_{1}\right) u d x=0 .
\end{aligned}
$$

Thus $E_{1}$ and $E_{2}$ are $\langle,\rangle_{\gamma}$ orthogonal. Thus the splitting is algebraic. Since $E_{2}$ is the image of a continuous linear map and $E_{1}$ is closed, $E_{2}$ is closed and the sum is topological (see, e.g.: [2], Lemma 4.5).

In a neighborhood of $\gamma$ any $g$ can thus be uniquely written $g=\gamma+h_{1}+h_{2}$ where $h_{i} \in E_{i}$. Consider the mapping $\psi$ of a neighborhood of zero in $E_{1} \oplus E_{2}$ to $\mathscr{X}_{s-1, \delta+1}^{p}$ (vector fields of type $\left.M_{s-1, \delta+1}^{p}\right)$ by $\left(h_{1}, h_{2}\right) \rightarrow\left(\gamma+h_{1}+h_{2}\right) \cdot \Gamma\left(\gamma+h_{1}+h_{2}\right)$. By the earlier properties of Sobolev spaces, $\psi$ is clearly $C^{\infty}$. The partial derivative with respect to $h_{2}$ at $(0,0)$ is the map $E_{2} \rightarrow \mathscr{X}_{s-1, \delta-1}^{p}$ given by $h_{2} \rightarrow \delta_{\gamma} h_{2}-\frac{1}{2} d \operatorname{tr}_{\gamma} h_{2}$. This map has kernel zero since $E_{1}$ and $E_{2}$ are $\langle,\rangle_{E}$ orthogonal and the map is onto since given $a_{j} \in \mathscr{X}_{s-1, \delta+1}^{p}$, define $u_{j}=-2 a_{j}+\frac{2}{3} \partial_{j} \Delta_{\gamma}^{-1} \partial_{i} a_{i}$ as above and let $h_{2}$ be as in the definition of $E_{2}$. Then $\delta_{\gamma} h_{2}-\frac{1}{2} d \operatorname{tr}_{\gamma} h_{2}=a$ as in the above calculation.

Thus, by the implicit function theorem ( $\psi$ is a submersion), $S$ is a manifold near $\gamma$.

The same type of reasoning will prove that near $\gamma, \mathscr{C} \cap S$ is a submanifold of $\mathscr{M}_{s, \delta}^{p}$. However, since we shall need the $\langle,\rangle_{g}$ orthogonal decomposition for the subspace $T_{g}(\mathscr{C} \cap S)$ explicitly, we shall give some additional details. 
4.2. Lemma. There is a neighborhood $V$ of $\gamma$ in $\mathscr{M}_{s, \delta}^{p}$ such that for $g \in V, S_{s, \delta}^{p}$ admits the $\langle,\rangle_{g}$ orthogonal decomposition as a topological sum

$$
S_{s, \delta}^{p}=B_{1}(g) \oplus B_{2}(g)
$$

where

$$
\begin{aligned}
B_{1}(g)= & \left\{h_{1} \in S_{s, \delta}^{p} \mid A_{g}\left(h_{1}\right) \equiv-\Delta_{g} \operatorname{tr}_{g} h_{1}+\delta_{g} \delta_{g} h_{1}\right. \\
& \left.-\operatorname{Ric}(g) \cdot h_{1}=0 \text { and } a_{g}\left(h_{1}\right) \equiv \delta_{g} h_{1}-\frac{1}{2} d \operatorname{tr}_{g} h_{1}-h_{1} \cdot \Gamma(g)=0\right\}
\end{aligned}
$$

and

$$
B_{2}(g)=\left\{h_{2} \in S_{s, \delta}^{p} \mid \quad \text { there is a } \quad U \in M_{s, \delta}^{p} \quad \text { and } \quad u \in \mathscr{X}_{s-1, \delta+1}^{p}\right.
$$

such that

$$
\begin{aligned}
h_{2} \equiv & h_{2}(U, u)=-g U+\Delta_{g}^{-1}\left(\operatorname{Hess}_{g} U-\operatorname{Ric}(g) U\right) \\
& \left.-\frac{1}{2} \Delta_{g}^{-1}\left(L_{u} g-g \delta_{g} u\right)-\Delta_{g}^{-1}(\Gamma(g) \cdot u)\right\} .
\end{aligned}
$$

In fact, $\mathscr{C} \cap S \cap V$ is a submanifold of $\mathscr{M}_{s, \delta}^{p}$ with tangent space given by

$$
T_{g}(\mathscr{C} \cap S)=B_{1}(g)
$$

i.e., $h \in T_{g} \mathscr{C} \cap S$ iff

and

$$
\begin{aligned}
& -\Delta_{g} \operatorname{tr}_{g} h+\delta \delta h-\operatorname{Ric}(g) \cdot h=0 \\
& \delta_{g} h-\frac{1}{2} d\left(\operatorname{tr}_{g} h\right)-h \cdot \Gamma(g)=0 .
\end{aligned}
$$

Proof. First of all we note that $B_{1}(g)$ and $B_{2}(g)$ are $\langle,\rangle_{g}$ orthogonal. Let $h_{1} \in B_{1}(g)$ and $h_{2} \in B_{2}(g)$. Then

$$
\begin{aligned}
\left\langle h_{1}, h_{2}\right\rangle_{g}= & \int_{\mathbb{R}^{3}} \nabla_{g} h_{1} \cdot \nabla_{g} h_{2} d \mu(g) \\
= & -\int_{\mathbb{R}^{3}} h_{1} \cdot \Delta_{g} h_{2} d \mu(g) \\
= & -\int_{\mathbb{R}^{3}}\left\{-\operatorname{tr} h_{1} \Delta_{g} U+h_{1} \cdot \operatorname{Hess}_{g} U-h_{1} \cdot \operatorname{Ric}(g) U\right. \\
& \left.-\frac{1}{2}\left(h_{1} \cdot L_{u} g-\operatorname{tr}_{g} h_{1} \cdot \delta_{g} u\right)-h_{1} \cdot(\Gamma(g) \cdot u)\right\} d \mu(g) \\
= & -\int_{\mathbb{R}^{3}}\left\{A_{g}\left(h_{1}\right) U+a_{g}\left(h_{1}\right) \cdot u\right\} d \mu(g) \\
= & 0 .
\end{aligned}
$$

Now define for $g \in \mathscr{M}_{s, \delta}^{p}$ the linear mapping

$$
\begin{aligned}
& \Lambda_{g}: M_{s, \delta}^{p} \times \mathscr{X}_{s-1, \delta+1}^{p} \rightarrow M_{s-2, \delta+2}^{p} \times \mathscr{X}_{s-1, \delta+1}^{p} \\
& \Lambda_{g}(U, u)=\left(L_{g}(U, u), l_{g}(U, u)\right)
\end{aligned}
$$

where

$$
\begin{aligned}
L_{g}(U, u)= & \Delta_{g} U+\nabla^{j} \nabla^{i} \Delta_{g}^{-1}\left(\nabla_{i} \partial_{j} U\right)-\nabla^{j} \nabla^{i}\left(\Delta_{g}^{-1} R_{i j} U\right) \\
& -\nabla^{j} \nabla^{i} \Delta_{g}^{-1}\left(\frac{1}{2} \nabla_{i} u_{j}+\frac{1}{2} \nabla_{j} u_{i}-\Gamma_{i j}^{k} u_{k}\right)
\end{aligned}
$$

and

$$
\begin{aligned}
\left(l_{g}(U, u)\right)_{j}= & \nabla^{i} \Delta_{g}^{-1}\left(\nabla_{i} \partial_{j} U-R_{i j} U-\frac{1}{2} \nabla_{i} u_{j}\right. \\
& \left.-\frac{1}{2} \nabla_{j} u_{i}\right)-\nabla^{i} \Delta_{g}^{-1}\left(\Gamma_{i j}^{k} u_{k}\right)+\frac{1}{4} \partial_{j} \Delta_{g}^{-1} \nabla_{l} u^{l} \\
& -\left(\Gamma \cdot h_{2}(U, u)\right)_{j}
\end{aligned}
$$


As in [18], $\S 3$, one sees that $\Lambda_{g}$ is a $C^{\infty}$ function of $g$ with values in the Banach space

$$
\mathscr{L}\left(M_{s, \delta}^{p} \times \mathscr{X}_{s-1, \delta+1}^{p}, M_{s-2, \delta+2}^{p} \times \mathscr{X}_{s-1, \delta+1}^{p}\right)
$$

of continuous linear maps.

The maps $L, l$ are defined so that

$$
A_{g}\left(h_{2}\right)=L_{g}(U, u) \text { and } a_{g}\left(h_{2}\right)=l_{g}(U, u)
$$

where $h_{2}=h_{2}(U, u)$ is given in the definition of $B_{2}(g)$. This can be verified by a straightforward calculation.

For $g=\gamma$ we have

$$
L_{\gamma}(U, u)=2 \Delta_{\gamma} U-\partial_{i} u_{i}
$$

and

$$
\left(l_{\gamma}(U, u)\right)_{j}=\partial_{j} U-\frac{1}{2} u_{j}-\frac{1}{4} \partial_{j} \Delta_{\gamma}^{-1} \partial_{i} u_{i} .
$$

Since $L_{\gamma}(U, u)=F$ and $l_{\gamma}(U, u)=f$ have a unique solution, namely

$$
U=\Delta_{\gamma}^{-1}\left(\frac{3}{2} F-2 \delta f\right)
$$

and

$$
u_{j}=2\left\{\partial_{j} \Delta_{\gamma}^{-1} F-\partial_{j} \Delta_{\gamma}^{-1} \delta f-f_{j}\right\}
$$

we see that $\Lambda_{\gamma}$ is an isomorphism.

Therefore, since the isomorphisms are open, there is a neighborhood $V$ of $\gamma$ such that for $g \in V, \Lambda_{g}$ is an isomorphism.

Given $h \in S_{s, \delta}^{p}, g \in V$, let $(U, u)=\Lambda_{g}^{-1}\left(A_{g}(h), a_{g}(h)\right)$ and $h_{2}=h_{2}(U, u) \in B_{2}(g)$. Then $h_{1}=h-h_{2}$ clearly belongs to $B_{1}(g)$, since $A_{g}\left(h_{1}\right)=A_{g}(h)-A_{g}\left(h_{2}\right)=L_{g}(U, u)-$ $A_{g}\left(h_{2}\right)=0$ and similarly, $a_{g}\left(h_{1}\right)=0$.

Thus we have an algebraic splitting and, as in Lemma 4.1, the splitting is then topological as well.

Finally, we consider the map

$$
\begin{aligned}
& \Sigma: \mathscr{M}_{s, \delta}^{p} \rightarrow M_{s-2, \delta+2}^{p} \times \mathscr{X}_{s-1, \delta+1}^{p} \\
& \Sigma(g)=(R(g), g \cdot \Gamma) .
\end{aligned}
$$

As above, $\Sigma(g)$ is $C^{\infty}$ and has derivative $D \Sigma(g)$ given by

$$
\begin{aligned}
& D \Sigma(g)=0 \quad \text { on } \quad B_{1}(g) \\
& D \Sigma(g)=\left(A_{g}, a_{g}\right) \text { on } \quad B_{2}(g) .
\end{aligned}
$$

Therefore, by the above, $D \Sigma(g)$ is an isomorphism on $B_{2}(g)$ for $g \in V$. The lemma therefore follows.

This argument also contains the proof that $\mathscr{C}$ is a manifold near $\gamma([13,18])$. In the sequel, $\mathscr{C} \cap S$ will stand for $\mathscr{C} \cap S \cap V$.

4.3. Theorem ${ }^{1}$. There exists an $\varepsilon>0$ such that for $g \in \mathscr{C} \cap S,\|g-\gamma\|_{M_{s, o}^{p}}<\varepsilon$ and $g \neq \gamma, m(g)>0$. In fact, $m(g) \geqq C\|g-\gamma\|_{E}^{2}$ for a constant $C>0$.

This result can also be proved using the Morse lemma on Banach manifolds, in the formulation given by Tromba [28] (see also [18], Lemma 5, p. 543). However, it seems simpler to give a direct proof. 
Proof. Let $V_{\varepsilon}=\left\{g \in \mathscr{C} \cap S \mid\|g-\gamma\|_{M_{s, \delta}^{p}}<\varepsilon\right\}$ and let $g(t)$ be a smooth curve in $V_{\varepsilon}$ joining $\gamma$ to $g \in V_{\varepsilon}, 0 \leqq t \leqq 1$. Since $\gamma$ is a critical point of $m, m(g)=m^{\prime \prime}(g(\tau)) / 2$ for some $\tau, 0<\tau<1$. However, if we let $h=g^{\prime}(t)$ and $k=g^{\prime \prime}(t)$, then

$$
m^{\prime \prime}(g(t))=d^{2} \bar{m}(g(t))(h, h)+d \bar{m}(g(t)) \cdot k .
$$

Since $g(t) \in \mathscr{C} \cap S, h$ satisfies (4.1). From 3.1, writing $\int-\frac{1}{2}(d \operatorname{tr} h)^{2} d \mu(g)=\int \frac{1}{2}(\operatorname{tr} h)$. $\Lambda(\operatorname{tr} h) d \mu(g)$, using (4.1) and replacing $\delta h$ by $\frac{1}{2} d \operatorname{tr} h-h \cdot \Gamma$ from 4.1, we get:

$$
\begin{aligned}
m^{\prime \prime}(g(t))= & \int_{\mathbb{R}^{3}}\left\{\frac{1}{2} \nabla h \cdot \nabla h-\frac{1}{2} \operatorname{tr} h \operatorname{Ric}(g) \cdot h+\operatorname{Ric}(g) \cdot(h \times h)\right. \\
& \left.+\frac{1}{2} d(\operatorname{tr} h) \cdot(h \cdot \Gamma)-(h \cdot \Gamma)(h \cdot \Gamma)+\operatorname{Ric}(g) \cdot k\right\} d \mu(g) .
\end{aligned}
$$

In order to estimate (4.2) we use the following estimate noted in Lemma 1.3.

$$
\|f\|_{L^{\prime}} \leqq C\|f\|_{E}
$$

from which we get, by Hölder's inequality,

$$
\left|\int_{\mathbb{R}^{3}} f^{2} \varrho d^{3} x\right| \leqq\|\varrho\|_{L^{3 / 2}}\|f\|_{L^{6}}^{2} \leqq C\|\varrho\|_{L^{3 / 2}}\|f\|_{E}^{2} .
$$

The following lemma estimates the Ricci tensor:

4.4. Lemma. If $g \in \mathscr{M}_{s, \delta}^{p}$ and $\varrho=\operatorname{Ric}(g)$, then

$$
\|\varrho\|_{L^{3 / 2}} \leqq C\|g-\gamma\|_{M_{s, \delta}^{p}} .
$$

Proof. One uses the explicit expression for $\operatorname{Ric}(g)=R_{i j}, R_{i j}=\frac{1}{2} g^{k l}\left\{-\partial_{k l}^{2} g_{i j}-\right.$ $\left.\partial_{i j}^{2} g_{k l}+\partial_{k j}^{2} g_{i l}+\partial_{i l}^{2} g_{k j}\right\}-g_{k l} \Gamma_{i j}^{k}(g \cdot \Gamma)^{l}+g^{k l} g_{m n} \Gamma_{i k}^{m} \Gamma_{l j}^{n}$. The terms in braces can be estimated as follows:

$$
\int\left|g^{i j} \partial_{h k}^{2} g_{l m}\right|^{3 / 2} d^{3} x \leqq \sup \left|g^{i j}\right|^{3 / 2} \int\left|\partial_{h k}^{2} g_{l m}\right|^{3 / 2} d^{3} x .
$$

Let $f=\partial_{h k}^{2} g_{i j} \in M_{s-2, \delta+2}^{p}$, so that with $q=\frac{2 p}{3}, q^{\prime}=\frac{2 p}{2 p-3}$, Hölder's inequality gives

$$
\int|f|^{3 / 2} d^{3} x \leqq\left\{\int \psi^{q}|f|^{3 q / 2} d^{3} x\right\}^{1 / q}\left\{\int \psi^{-q^{\prime}} d^{3} x\right\}^{1 / q^{\prime}} .
$$

If we choose $\psi(x)=\left(1+|x|^{2}\right)^{3 / 2}$, we get

$$
\int|f|^{3 / 2} d^{3} x \leqq C\left\{\int \sigma^{2 p}|f|^{p} d^{3} x\right\}^{1 / q}
$$

since $\sigma(x)=\sqrt{1+|x|^{2}}$, and $\int \frac{d^{3} x}{\left(1+x^{2}\right)^{3 q^{\prime} / 2}}<\infty$ if $q^{\prime}>1$. Therefore,

$$
\|f\|_{L^{3 / 2}} \leqq C\left\|\sigma^{2} f\right\|_{L^{p}} \leqq C\|g-\gamma\|_{M_{s, \delta}^{p},}
$$

from the definition of the $M_{s, \delta}^{p}$ norm.

Since $\|g-\gamma\|_{C^{0}} \leqq C\|g-\gamma\|_{\mathcal{M}_{s, \delta}^{p}}$, we have the required estimate on the terms in braces. For the remaining terms, we must estimate $\left\|\partial_{i} g_{k l} \partial_{j} g_{m n}\right\|_{L^{3 / 2}}$. Taking $f=\partial_{i} g_{k l} \in M_{s-1, \delta+1}^{p}$, and $\phi=\partial_{j} g_{m n} \in M_{s-1, \delta+1}^{p}$, we have (using $|x y| \leqq \frac{1}{2}\left(|x|^{2}+|y|^{2}\right.$ )

$$
\begin{aligned}
\int|f \phi|^{3 / 2} d^{3} x \leqq & \frac{1}{2}\left(\int|f|^{3} d^{3} x+\int|\phi|^{3} d^{3} x\right) \\
\leqq & \leqq \\
2 & \left(\int \sigma^{3 q}|f|^{3 q} d^{3} x\right)^{1 / q}\left(\int \sigma^{-3 q^{\prime}} d^{3} x\right)^{1 / q^{\prime}} \\
& +\frac{1}{2}\left(\int \sigma^{3 q}|\phi|^{3 q} d^{3} x\right)^{1 / q}\left(\int \sigma^{-3 q^{\prime}} d^{3} x\right)^{1 / q^{\prime}}
\end{aligned}
$$


Now we choose $q=p / 3$ to arrive at, as above,

$$
\begin{aligned}
\int|f \phi|^{3 / 2} d^{3} x & \leqq C\left(\|\sigma f\|_{L^{p}}+\|\sigma \phi\|_{L^{p}}\right) \\
& \leqq C^{\prime}\|g-\gamma\|_{M_{s, \delta}^{p}} .
\end{aligned}
$$

These estimates prove Lemma 4.4 .

Combining (4.4) and (4.5), we see that the second and third terms of (4.2) are $\leqq C\|g-\gamma\|_{M_{s, \delta}^{p}}\|h\|_{E}^{2}$. The fourth and fifth terms can be similarly estimated, and $\int \nabla h \cdot \nabla h d \mu(g)$ differs from $\|h\|_{E}^{2}$ by terms of the same sort.

Lemma 4.2 proved that $\langle,\rangle_{g}$ has a smooth orthogonal projection

$$
P_{g}: T_{g} \mathscr{M}_{s, \delta}^{p} \rightarrow T_{g}(\mathscr{C} \cap S), g \in \mathscr{C} \cap S .
$$

Combining this with 3.2 , we get (see $[15,16])$ :

4.5. Lemma. The weak Riemannian structure induced on $\mathscr{C} \cap S$ by $\langle,\rangle_{g}$ has a smooth connection.

If $g(t)$ is a geodesic curve for $\langle,\rangle_{g}$, then as the connection is smooth in the $M_{s, \delta}^{p}$ topology we can shrink $V_{\varepsilon}$ to assure that $g(t) \in V_{\varepsilon}$, i.e., there is a $\langle,\rangle_{g}$ normal coordinate neighborhood about $\gamma$ which lies in the $M_{s, \delta}^{p} \varepsilon$-ball. Therefore, $\|h\|_{E}$ is equivalent to $\|g-\gamma\|_{E}$ or the $E$-distance from $g$ to $\gamma$, in a neighborhood of $\gamma$.

We shall need to expand on these points somewhat. A geodesic $g(t)$ for $\langle,\rangle_{g}$ on $\mathscr{C} \cap S$ satisfies the equation obtained by differentiating $\Sigma(g(t))=0$ twice:

$$
D \Sigma(g) \frac{d^{2} g}{d t^{2}}+D^{2} \Sigma(g)\left(\frac{d g}{d t}, \frac{d g}{d t}\right)=0
$$

with $g(t) \in \mathscr{C} \cap S, \frac{d g}{d t} \in B_{1}(g)$. In the usual way, the acceleration is orthogonal to $\mathscr{C} \cap S: \frac{d^{2} g}{d t^{2}} \in B_{2}(g)$.

Recall that $\Sigma(g)=(R(g), g \cdot \Gamma(g)), D \Sigma(g) \in \mathscr{L}\left(S_{s, \delta}^{p}, M_{s-2, \delta+2}^{p} \times \mathscr{X}_{s-1, \delta+1}^{p}\right)$ and $D^{2} \Sigma(g) \in \mathscr{L}^{2}\left(S_{s, \delta}^{p}, M_{s-2, \delta+2}^{p} \times \mathscr{X}_{s-1, \delta+1}^{p}\right)$ (the continuous bilinear mappings).

We shall use these remarks to prove the following, which deals with the last term of $m^{\prime \prime}$.

4.6. Lemma. If $g(t)$ is a geodesic in $\mathscr{C} \cap S$ from $\gamma$ to $g, h=\frac{d g}{d t}$ and $k=\frac{d^{2} g}{d t^{2}}$, then

$$
\left|\int_{\mathbb{R}^{3}} \operatorname{Ric}(g) \cdot k d \mu(g)\right| \leqq C\|g-\gamma\|_{S_{s, \delta}^{p}}\|h\|_{E}^{2} .
$$

Proof. Since $k \in B_{2}(g)$ we can write

$$
\begin{aligned}
k= & -g V+\Delta_{g}^{-1}\left(\operatorname{Hess}_{g} V-\operatorname{Ric}(g) V\right. \\
& \left.-\frac{1}{2} L_{v} g+\frac{1}{2} g \delta_{g} v-\Gamma(g) \cdot v\right)
\end{aligned}
$$


for $V \in M_{s, \delta}^{p}$ and $v \in \mathscr{X}_{s-1, \delta+1}^{p}$. Using $R(g)=0$, we have:

$$
\begin{aligned}
I & \equiv \int_{\mathbb{R}^{3}} \operatorname{Ric}(g) \cdot k d \mu(g) \\
& =\int_{\mathbb{R}^{3}} \Delta_{g}^{-1} \operatorname{Ric}(g) \Delta_{g} k d \mu(g) \\
& =\int_{\mathbb{R}^{3}}(\lambda V+\mu v) d \mu(g)
\end{aligned}
$$

where $\lambda=\nabla_{i} \nabla_{j} \Delta_{g}^{-1} R^{i j}-R_{i j} \Delta_{g}^{-1} R^{i j}$ and $\mu^{j}=\nabla_{i} \Delta_{g}^{-1} R^{i j}-\Gamma_{l m}^{j} \Delta_{g}^{-1} R^{l m}$.

By Lemma 4.2 and the remarks above, $(V, v)=\Lambda_{g}^{-1}\left(A_{g}(k), a_{g}(k)\right)=$ $\Lambda_{g}^{-1}(D \Sigma(g) \cdot k)=\Lambda_{g}^{-1}\left(D^{2} \Sigma(g)(h, h)\right)=\Lambda_{g}^{-1}\left(D_{g} A_{g}(h) \cdot h, D_{g} a_{g}(h) \cdot h\right)$. Let $F=D_{g} A_{g}(h) \cdot h$ and $f=D_{g} a_{g}(h) \cdot h$, and let $\bar{M}_{s, \delta}^{p}$ denote functions whose difference with a constant is in $M_{s, \delta}^{p}$. From the expressions for $A_{g}$ and $a_{g}$ (see Lemma 4.2), $F$ is a linear combination of terms $h \cdot \partial^{2} h, \partial h \cdot \partial h, h \cdot \partial h$ and $h \cdot h$ with coefficients (depending on $g$ ) in $\bar{M}_{s, \delta}^{p}, \bar{M}_{s, \delta}^{p}, M_{s-1, \delta+1}^{p}$ and $M_{s-2, \delta+2}^{p}$ respectively. Similarly, $f$ is a linear combination of terms $h \cdot \partial h$, and $h \cdot h$ with coefficients in $\bar{M}_{s, \delta}^{p}$ and $M_{s-1, \delta+1}^{p}$ respectively.

Write $^{2}$

$$
\begin{aligned}
I & =\int_{\mathbb{R}^{3}}(\lambda, \mu) \cdot\left(\Lambda_{g}^{-1}(F, f)\right) d \mu(g) \\
& =\int_{\mathbb{R}^{3}}\left(\left(\Lambda_{g}^{*}\right)^{-1}(\lambda, \mu)\right) \cdot(F, f) d \mu(g) .
\end{aligned}
$$

The function $\left(\Lambda_{g}^{*}\right)^{-1}(\lambda, \mu)$ is a smooth function in $M_{s, \delta}^{p}$ of $g \in \mathscr{M}_{s, \delta}^{p}$. Then the terms from $F$ involving $\partial h \cdot \partial h$, for example, have the form

$\int_{\mathbb{R}^{3}} \varrho(g) \partial h \cdot \partial h d x$

for $\varrho(g) \in M_{s, \delta}^{p}, \varrho(\gamma)=0, \varrho(g)$ depending smoothly on $g$. Since $\|\varrho(g)\|_{L^{\infty}} \leqq C\|g-\gamma\|_{M_{s, \delta}^{p}}$ we get a bound of the form $C\|g-\gamma\|_{M_{s, o}^{p}}\|h\|_{E}^{2}$. Similarly, terms from $F$ or $f$ of the form $\int_{\mathbb{R}^{3}} \varrho(g) h \cdot \partial h d x$ are bounded by $\|h\|_{L^{6}}\|h\|_{E}\|\varrho(g)\|_{L^{6}} \leqq C\|h\|_{E}^{2}\|\varrho(g)\|_{M_{s-1, \delta}^{p}+1}$

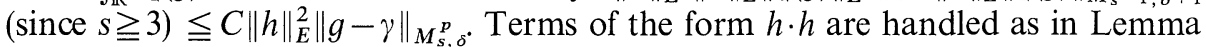
4.4 and terms involving $h \cdot \partial^{2} h$ are reduced to those just treated by integration by parts. This yields Lemma 4.6.

Theorem 1 foliows, for putting these inequalities together,

$$
\left|m^{\prime \prime}(g(t))-1 / 2\|h\|_{E}^{2}\right| \leqq C\|g-\gamma\|_{\mathcal{M}_{s, \delta}^{p}}\|h\|_{E}^{2}
$$

so if $\|g-\gamma\|_{M_{s, \delta}^{p}}$ is small enough,

$$
m^{\prime \prime}(g(t)) \geqq C^{\prime}\|h\|_{E}^{2} \geqq C\|g-\gamma\|_{E}^{2} .
$$

\section{Local Positivity of $m$}

To show $m>0$ in an $M_{s, \delta}^{p}$ neighborhood of $\gamma$, we shall actually show $S$ is a slice in the sense of Ebin and Palais (see [15]), using the action of $\overline{\mathscr{D}}_{s+1, \delta-1}^{p}$.

5.1. Theorem. There is an $M_{s, \delta}^{p}$ neighborhood of $\gamma$ in which $m>0 ; m=0$ only on metrices isometric to $\gamma$.

It suffices, by 4.2 , to prove:

2 The adjoint here is taken, in the usual $L_{2}$ sense and exists since $\Lambda_{g}$ is a pseudodifferential operator. It can be calculated explicitly from the expression for $\Lambda_{g}$ 
5.2. Lemma. If $\varepsilon$ is sufficiently small and $\|g-\gamma\|_{M_{s, \delta}}<\varepsilon$, then $\phi^{*} g \in S$ for some $\phi \in \overline{\mathscr{D}}_{s+1, \delta-1}^{p}$.

Proof. Let, in Euclidean coordinates, $\phi$ have components $x^{i}+f^{i}\left(x^{j}\right)$. Then $\phi^{*} g \in S$ if $\Delta_{g} \phi^{i}=0$, i.e., $\Delta_{g} f^{i}=\Gamma_{j k}^{i} g^{j k}$. Now $\Gamma_{j k}^{i} g^{j k}$ is not in $M_{s-2, \delta+2}^{p}$ necessarily, so we cannot yet apply $\Delta_{g}^{-1}$. However, we can differentiate, letting $F^{i}$ be the differential of $f^{i}$, so $F^{i}$ is a one form, and denoting by $\tilde{\Delta}_{g}$ the Lapalce-DeRham operator on forms, to obtain ${ }^{3}$

$$
\tilde{\Delta}_{g} F^{i}=d H^{i}
$$

where $H^{i}=\Gamma_{j k}^{i} g^{j k}$. Since $\tilde{\Delta}_{g}$ is an isomorphism of $M_{s, \delta}^{p}$ to $M_{s-2, \delta+2}^{p}$, there is a unique solution $F^{i} \in M_{s, \delta}^{p}$. However, since $s \geqq 3$ we can assert $\tilde{\Delta}_{g} d F^{i}=d \tilde{\Delta}_{g} F^{i}=$ $d\left(d H^{i}\right)=0$, so $d F^{i}=0$. Thus $F^{i}=\mathrm{d} f^{i}$ for some $f^{i}$. (Explicitly, we can choose

$$
f^{i}(x)=\int_{0}^{1} F^{i}(t x) \cdot x d t
$$

from the proof of the Poincare lemma.) Since $F^{i} \in M_{s, \delta}^{p}$ we see that $d f^{i} \in M_{s, \delta}^{p}$, so for $g^{j k} \Gamma_{j k}^{i}$ small in $M_{s-1, \delta+1}^{p}, d f^{i}$ will be small in $M_{s, \delta}^{p}$ so $\phi$ will be a $C^{1}$ diffeomorphism. Thus $\phi \in \mathscr{\mathscr { D }}_{s+1, \delta-1}^{p}$.

Remark. The coordinate invariance and the slice problem are discussed in an interesting manner in [9] using the ideas of York (see [22]). This might lead to an alternative method to that here.

One can deduce a number of corollaries which are implicit in the literature cited. For example (see [1]), if $g \in \mathscr{M}_{s, \delta}^{p}, \phi \in M_{s, \delta}^{p}+\{1\}$ and $\Delta_{g} \phi+8 R(g) \phi=0$ (so that $\left.R\left(\phi^{4} g\right)=0\right)$, then if $\phi^{4} g$ is near flat space, but $\phi^{4} g \neq \mathcal{O}_{\gamma}, \phi^{4} g-\gamma$ cannot fall off faster than $1 / r$ at $\infty$, since the mass of $\phi^{4} g$ is positive.

\section{Remarks on Global Positivity}

Let $Y$ be the $\langle$,$\rangle gradient of -m$ on $\mathscr{C}$. It is the projection on $\mathscr{C}$ of $-\operatorname{grad} \bar{m}=$ $\Delta_{g}^{-1} \operatorname{Ric}(g)(\text { see } 4.1,4.2)^{4}$. Then $Y$ is a smooth vector field on $\mathscr{C}$, in the $M_{s, \delta}^{p}$ topology.

From the above work, it follows that $\mathcal{O}_{\gamma}$ is an attracting set for $Y$; i.e., there is a neighborhood $U$ of $\mathcal{O}_{\gamma}$ such that if $g \in U$, the $Y$-trajectory of $g$ tends to $\mathcal{O}_{\gamma}$ as $t \rightarrow \infty$.

If we could show $\|Y(g)\|_{E} \geqq \varepsilon>0$ for $g$ outside such a neighborhood $U$, then $m$ would be $>0$ globally. Indeed, it is not hard to see that $A=\{g \mid$ the $Y$ trajectory of $g \rightarrow \mathcal{O}_{\gamma}$ as $\left.t \rightarrow+\infty\right)$ is both open and closed in that case.

One can contemplate more sophisticated methods as well, such as the minimax principle (cf. Palais [25]); however, there seem to be serious technical obstacles. For example, we did not prove that there is an $\varepsilon>0$ such that along any curve $g(t)$ emanating from $\gamma, m$ must reach the value $\varepsilon$. Such a property would seem to be crucial for the use of the minimax principle.

${ }^{3} \phi$ is a harmonic map from $\left(\mathbb{R}^{3}, g\right)$ to $\left(\mathbb{R}^{3}, \gamma\right)$ so general invariant formulas from Eells and Sampson ([17]) are available. However, it is just as easy to proceed directly in this case

${ }_{4}$ One can also use $Y(g)_{i j}=\Delta^{-1} R_{i j}-\frac{1}{2} g_{i j} \Delta^{-1}\left(R_{h k} \Delta^{-1} R^{h k}-\nabla_{h} \nabla_{k} \Delta^{-1} R^{h k}\right)$ which is a pseudo-gradient: $d m \cdot Y=\left\langle\Delta^{-1} \operatorname{Ric}(g), \Delta^{-1} \operatorname{Ric}(g)\right\rangle_{g}>0$ if $\operatorname{Ric}(g) \neq 0$ 


\section{References}

1. Araki, H.: On weak time-symmetric gravitational waves. Ann. Phys. 7, 456-465 (1959)

2. Berger, M., Ebin, D.: Some decompositions of the space of symmetric tensors on a Riemannian manifolg. J. Diff. Geom. 3, 379-392 (1969)

3. Brill,D.: On the positive definite mass of the Bondi-Weber-Wheeler time-symmetric graviational waves. Ann. Phys. 7, 466-483 (1959)

4. Brill, D., Deser, S.: Variational methods and positive energy in general relativity. Ann. Phys. 50, 548-570 (1968)

5. Brill, D., Deser, S.: Instability of closed spaces in general relativity. Commun. math. Phys. 32, 291-304 (1973)

6. Cantor, M.: Spaces of functions with asymptotic conditions on $\mathbb{R}^{n}$, Indiana University Math. J. 24, 897-902 (1975)

7. Cantor, M.: Perfect fluid flows over $\mathbb{R}^{n}$ with asymptotic conditions. J. Funct. Ann. 18, $73-84$ (1975)

8. Cantor, M.: Growth of solutions of elliptic operators with nonconstant coefficients on $\mathbb{R}^{n}$ (preprint)

9. Cantor, M.: Some problems of global analysis on asymptotically simple manifolds (in preparation)

10. Cantor, M., Fischer,A., Marsden, J., O’Murchadha, N., York, J.: On the existence of maximal slices. Commun. math. Phys. 49, 187-190 (1976)

11. Choquet-Bruhat, Y.: Cauchy problem. In: Gravitation; an introduction to current research (L. Witten ed.). Chichester: Wiley 1962

12. Choquet-Bruhat, Y.: Sous-variétés maximales, ou à courbure constante, de variétés lorentziennes. C.R. Acad. Sci. 280, 169-171 (1975)

13. Choquet-Bruhat, Y., Deser, S.: On the stability of flat space. Ann. Phys. 81, 165-178 (1973)

14. DeWitt, B.: Quantum theory of gravity. Phys. Rev. 160, 1113-1148 (1967)

15. Ebin, D.: The manifold of Riemannian metrics. Proc. Symp. Pure Math. 15, $11-40$ (1970)

16. Ebin,D., Marsden, J.: Groups of diffeomorphisms and the motion of an incompressible fluid. Ann. Math. 92, 102-163 (1970)

17. Eells, J., Sampson, J.: Harmonic maps of riemannian manifolds. Am. J. Math. 86, 109-160 (1964)

18. Fischer, A., Marsden, J.: Deformations of the scalar curvature. Duke Math. J. 42, 519—547 (1975)

19. Fischer, A., Marsden,J.: Linearization stability of nonlinear partial differential equations. Proc. Symp. Pure Math. 27, 219-263 (1975)

20. Geroch, R.: General Relativity. Proc. Symp. Pure Math., Vol. 27 (part 2), $401-414$ (1975) see also J. Math. Phys. 13, 956 (1972)

21. Lichnerowicz,A.: Propagateurs et commutateurs en relativité générale. Publ. I.H.E.S. 10, 56 (1961)

22. O’Murchadha, N., York, J.W.: Gravitational energy. Phys. Rev. D. 10, 2345-2357 (1974)

23. Nirenberg, L.: On elliptic partial differential equations. Ann. Scuola. Norm. Sup. Pisa 13, 115-162 (1959)

24. Nirenberg, L., Walker,H.: The null space of elliptic partial differential operators in $\mathbb{R}^{n}$. J. Math. Ann. Appl. 47, 271-301 (1973)

25. Palais, R.: Critical point theory and the minimax principle. Proc. Symp. Pure Math. 15, 185-212 (1970)

26. Regge, T., Teitelboim, C.: Role of surface integrals in the Hamiltonian formulation of general relativity. Ann. Phys. 88, 286-318 (1974)

27. Robinson, D., Winicour, J.: Energy of gravitational shock waves. J. Math. Phys. 13, 1435-1441 (1972) see also Phys. Rev. 3, 840 (1971)

28. Tromba,A.: Almost Riemannian structures on Banach manifolds, the Morse lemma and the Darboux theorem. Can. J. Math. 28, 640-652 (1976)

29. Tromba, A.: A general approach to Morse theory. J. Diff. Geom. (to appear)

Communicated by J.Ehlers

Received December 3, 1975 